\title{
Saturated Output Tabletop X-ray Lasers
}

J. Dunn, A. L. Osterheld, J. Nilsen, J. R. Hunter, Y. Li, A.
Ya. Faenov, T. A. Pikuz, V. N. Shlyaptsev

This article was submitted to

7th Inernational Conference on X-Ray Lasers,

Saint-Malo, France

June 18-23, 2000

\section{December 1, 2000}

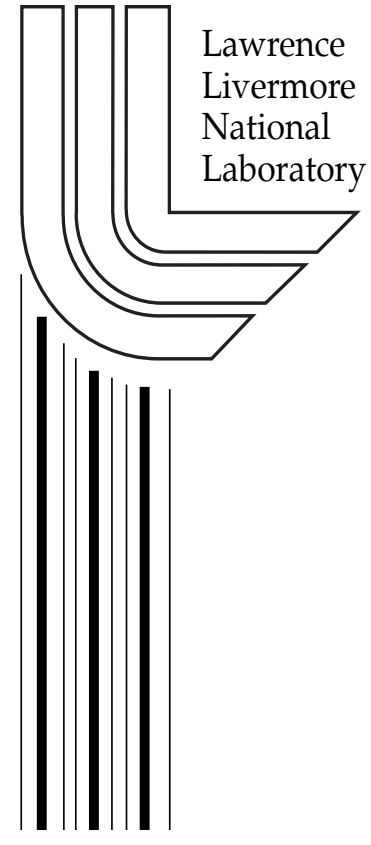




\section{DISCLAIMER}

This document was prepared as an account of work sponsored by an agency of the United States Government. Neither the United States Government nor the University of California nor any of their employees, makes any warranty, express or implied, or assumes any legal liability or responsibility for the accuracy, completeness, or usefulness of any information, apparatus, product, or process disclosed, or represents that its use would not infringe privately owned rights. Reference herein to any specific commercial product, process, or service by trade name, trademark, manufacturer, or otherwise, does not necessarily constitute or imply its endorsement, recommendation, or favoring by the United States Government or the University of California. The views and opinions of authors expressed herein do not necessarily state or reflect those of the United States Government or the University of California, and shall not be used for advertising or product endorsement purposes.

This is a preprint of a paper intended for publication in a journal or proceedings. Since changes may be made before publication, this preprint is made available with the understanding that it will not be cited or reproduced without the permission of the author.

This report has been reproduced directly from the best available copy.

Available to DOE and DOE contractors from the Office of Scientific and Technical Information

P.O. Box 62, Oak Ridge, TN 37831

Prices available from (423) 576-8401

http:/ / apollo.osti.gov/bridge/

Available to the public from the National Technical Information Service

U.S. Department of Commerce 5285 Port Royal Rd., Springfield, VA 22161

http://www.ntis.gov/

OR

Lawrence Livermore National Laboratory

Technical Information Department's Digital Library

http://www.llnl.gov/tid/Library.html 


\title{
Saturated Output Tabletop X-ray Lasers
}

\author{
James Dunn, Albert L. Osterheld, Joseph Nilsen, James R. Hunter, Yuelin Li ${ }^{1}$, \\ Anatoly Ya. Faenov ${ }^{2}$, Tatiana A. Pikuz ${ }^{2}$ and Vyacheslav N. Shlyaptsev ${ }^{3}$ \\ Lawrence Livermore National Laboratory, Livermore, CA 94550, USA \\ ${ }^{1}$ ILSA, Lawrence Livermore National Laboratory, Livermore, CA 94550, USA \\ ${ }^{2}$ MISDC of VNIIFTRI, Mendeleevo, Moscow Region, 141570, Russia \\ ${ }^{3}$ DAS, University of California Davis-Livermore, Livermore, CA 94550,USA \\ This work was performed under the auspices of the U.S. Department of Energy by \\ the University of California, Lawrence Livermore National Laboratory under \\ contract No. W-7405-Eng-48.
}

\begin{abstract}
The high efficiency method of transient collisional excitation has been successfully demonstrated for Ne-like and Ni-like ion x-ray laser schemes with small 5-10 J laser facilities. Our recent studies using the tabletop COMET (Compact Multipulse Terawatt) laser system at the Lawrence Livermore National Laboratory (LLNL) have produced several x-ray lasers operating in the saturation regime. Output energy of $10-15 \mu \mathrm{J}$ corresponding to a gL product of 18 has been achieved on the Ni-like $\mathrm{Pd} 4 \mathrm{~d} \rightarrow 4 \mathrm{p}$ transition at 147 $\AA$ with a total energy of $5-7 \mathrm{~J}$ in a $600 \mathrm{ps}$ pulse followed by a $1.2 \mathrm{ps}$ pulse. Analysis of the laser beam angular profile indicates that refraction plays an important role in the amplification and propagation process in the plasma column. We report further improvement in the extraction efficiency by varying a number of laser driver parameters. In particular, the duration of the second short pulse producing the inversion has an observed effect on the x-ray laser output.
\end{abstract}

\section{INTRODUCTION}

Gain saturation is the desired operating regime for x-ray lasers in order to optimize the extraction efficiency of energy from the plasma gain medium. In the early 1990s this was demonstrated for Nelike ion collisional excitation schemes for $3 p \rightarrow 3 s J=2 \rightarrow 1$ lasers of Se at $204 \AA$ and Ge at $232 \AA$ with $500 \mathrm{~J}$ to kilojoules of drive energy (see for example [1] and other work in referenced in [2]). The main challenge to improving x-ray lasers has been taking a tabletop scheme and succesfully driving it into saturation. There has been considerable difficulty in meeting this challenge by many promising schemes including optical field ionization and recombination [2]. Towards this goal, substantial improvement has been made in the last $5-7$ years in the reduction of the laser drive energy required to generate the population inversion by the robust collisional excitation scheme. This has allowed further progress towards high output, shorter wavelength $\mathrm{x}$-ray lasers. The prepulse technique [3] when used in conjunction with 75 ps pulses with 75 to $150 \mathrm{~J}$ energy has achieved gain saturation on various Ni-like $4 d \rightarrow 4 p J=0 \rightarrow 1$ transitions from $139 \AA$ to $59 \AA$ for $\mathrm{Ag}$ and Dy ions $[4,5]$. Recently, the laser drive energy required for saturated operation has been reduced further to $30 \mathrm{~J}$ for Ni-like $\mathrm{Pd} 4 d \rightarrow 4 p J=0 \rightarrow 1 \mathrm{x}$-ray lasers at $147 \AA$ [6].

Progress towards a tabletop $\mathrm{x}$-ray laser has advanced in parallel because the advantages of reduced size, low cost and high repetition rate are important for future development of applications in this field. The fast capillary discharge plasma operating at $469 \AA$ for Ne-like Ar has shown gain saturation and recently this has been extended to high repetition rate, high average power operation [7]. The transient collisional excitation scheme has been proposed to achieve tabletop operation for laser-driven schemes [8]. This utilizes two laser pulses where a long nanosecond pulse generates the plasma and creates the required closed shell Ne-like or Ni-like ionization conditions. After a delay to 
allow for plasma cooling and expansion which is desirable for both optimum pumping and ray propagation along the plasma column, a 1 ps laser pulse generates a transient population inversion. This fast heating timescale allows efficient pumping without perturbing the ionization. Very high $\mathrm{x}-$ ray laser gains greater than $100 \mathrm{~cm}^{-1}$ are predicted and so saturation for target lengths of less than 1 $\mathrm{cm}$ is possible. The advantage with this scheme is that less than $5-10 \mathrm{~J}$ of laser energy from a chirped pulse amplification (CPA) tabletop laser is sufficient to drive the inversion. The initial demonstration of the transient scheme was shown for Ne-like Ti $3 p \rightarrow 3 s$ transition at $326 \AA$ [9] was a landmark for this type of collisional $\mathrm{x}$-ray laser. The scheme was extended to the Ni-like ion sequence for the $\operatorname{Pd} 4 d \rightarrow 4 p$ line at $146.8 \AA$ [10]. Further work on Transient gain saturation has also been demonstrated on Ne-like Ti at $326 \AA$ and Ge at $196 \AA$ using the larger Vulcan-CPA laser at the Rutherford Appleton Laboratory [11]. This intense experimental activity in many laboratories including France, Japan, Germany and the United Kingdom, over the last $2-3$ years shows the rapid achievement of maturity for transient gain x-ray lasers. (For example, see work by Klisnick et al., Kato et al., Nickles et al., Tallents et al., in these proceedings [12]).

The rest of this paper is constructed as follows: we review the experimental setup for the gain saturation experiments on the COMET laser in the next section and report the experimental results with modeling for Ni-like $\mathrm{Pd}$. A description for the experimental results for gain saturation of $\mathrm{Ne}$ like $\mathrm{Cr}$ is given, while initial results of the short pulse duration study are presented.

This work was performed under the auspices of the U.S. Department of Energy by the University of California, Lawrence Livermore National Laboratory under contract No. W$7405-$ Eng -48 .

\section{EXPERIMENTAL DESCRIPTION}

The experiments were conducted on the COMET laser facility at LLNL [13]. This laser, operating at $1054 \mathrm{~nm}$ wavelength, utilizes the technique of CPA to produce three beams of nominally $500 \mathrm{fs}$ (compressed) or $600 \mathrm{ps}$ (FWHM) pulse duration with a repetition rate of 1 shot every 4 minutes. This shot rate is limited by the cool down time of the final $50 \mathrm{~mm}$ amplifier stages. The X-ray laser generation requires two laser pulses. The short pulse was lengthened to $1.0-1.5 \mathrm{ps}$ with energy of $4.5-5.5 \mathrm{~J}$ while the long pulse energy was typically $0.5 \mathrm{~J}$ to $3 \mathrm{~J}$ delivered in the line focus at the target chamber. The peak-to-peak delay between the laser pulses was found to be optimal at $700 \mathrm{ps}$ for the Ni-like ion X-ray lasers with the short pulse arriving after the long pulse. The line focus length of $1.1 \mathrm{~cm}$ was achieved with a cylindrical lens and an on-axis paraboloid.

The on axis X-ray laser output was observed with a 1200 line $\mathrm{mm}^{-1}$ variable-spaced flat-field grating spectrometer with a back-thinned $1024 \times 1024$ charge-coupled device (CCD). Filters giving attenuation between $10-30 \times$ were used to prevent the x-ray laser saturating the CCD. Fiducial wires were placed close to the spectrometer entrance slit and aligned to the target surface with a

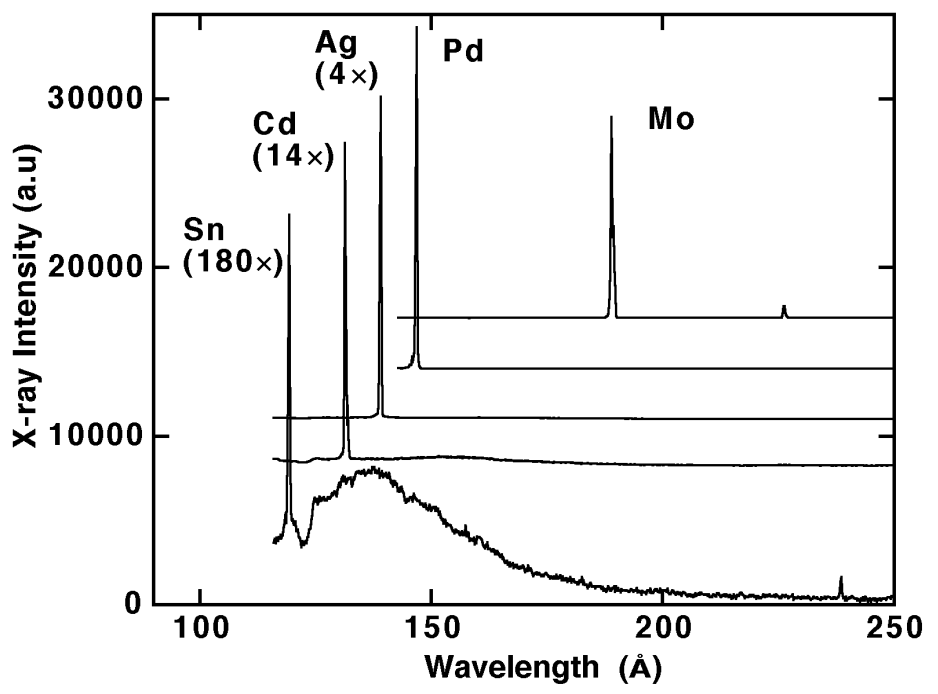

Figure 1. Ni-like ion sequence for $\mathrm{Mo}, \mathrm{Pd}, \mathrm{Ag}, \mathrm{Cd}$ and $\mathrm{Sn} . \mathrm{Ag}$, $\mathrm{Cd}$ and $\mathrm{Sn}$ have intensity multipliers of $4 \times, 14 \times$, and $180 \times$ respectively, to normalize the line intensity. telescope to calibrate the angular deflection and beam divergence of the $\mathrm{x}$ ray laser in the horizontal direction. Flat polished target slabs were used in the experiment and tilted back by $\sim 5 \mathrm{mrad}$ in the horizontal direction to compensate for refraction of the x-ray laser in the plasma column. A CCD x-ray slit camera with $25 \mu \mathrm{m}$ spatial resolution monitored the line focus plasma uniformity and overlap of the laser pulses. An on-axis Xray multilayer-coated imaging system spatially resolved the 2-dimensional $\mathrm{x}$ ray laser beam exit profile and output energy for the $189 \AA$ Ni-like Mo $4 d \rightarrow 4 p$ laser [14].

It should be mentioned that several changes were introduced to the 
experiment in comparison with our previous x-ray laser campaigns [10, 13]. The long pulse was defocused to a width of $\sim 150 \mu \mathrm{m}$ (FWHM) while the short pulse beam was focused to $80 \mu \mathrm{m}$. The main idea was to produce a more uniform lateral plasma medium and increase absorption prior to the excitation process driven by the picosecond laser. More importantly, a traveling wave scheme was introduced to mitigate against the reduced amplification at longer target lengths resulting from the short-lived transient gain lifetime and increase the laser output. A simple reflection echelon technique was adopted to produce the traveling wave as described in previously related x-ray laser work [15]. The traveling wave optic consisted of five flat mirror segments placed before the focusing optics. This optic in fact produces five separate line foci at the target which are carefully aligned using an imaging system. Each mirror segment was offset by $0.12 \mathrm{~cm}$ to introduce a traveling wave towards the spectrometer with a delay of 7.7 ps per step. This corresponded to a phase velocity of $c$ along the line focus length, matched to the propagation of the x-ray laser in the gain region. This was essential to generate small signal gain saturation in Pd, described in the next section [16]. For nontraveling wave excitation the reflection echelon was replaced with a flat mirror. Optical streak camera measurements at the line focus with the short pulse beam indicated that a lower limit of $\sim 11 \mathrm{c}$ was produced with the flat mirror effectively creating simultaneous excitation along the line focus.

\section{NI-LIKE PALLADIUM SATURATION RESULTS}

Detailed descriptions of small signal gain saturation for a Ni-like Mo x-ray laser together with spatial imaging of the gain region can be found in [14]. This work concluded that the gain length product of $16.8 \pm 0.6$ was determined indicating that the laser intensity was several times above the saturation regime. Here, we will describe the results primarily for Pd. As a comparison, Figure 1 shows the spectra for several Ni-like ion sequence $\mathrm{x}$-ray lasers for $0.9 \mathrm{~cm}$ targets of $\mathrm{Mo}(\mathrm{Z}=42), \mathrm{Pd}$ $(Z=46), \operatorname{Ag}(Z=47), C d(Z=48)$ and $\mathrm{Sn}(Z=50)$. These targets were irradiated with a $1.2 \pm 0.5 \mathrm{~J}, 600$ ps laser pulse followed by $5.0 \pm 0.3 \mathrm{~J}, 1 \mathrm{ps}$ pulse with a delay of $700 \mathrm{ps}$. The spectra were obtained with the 5-segment traveling wave reflection echelon optic. The $4 d \rightarrow 4 p$ x-ray laser transitions are dominant in each spectrum with the highest intensity observed for the $188.9 \AA, 146.8 \AA$ and $138.9 \AA$

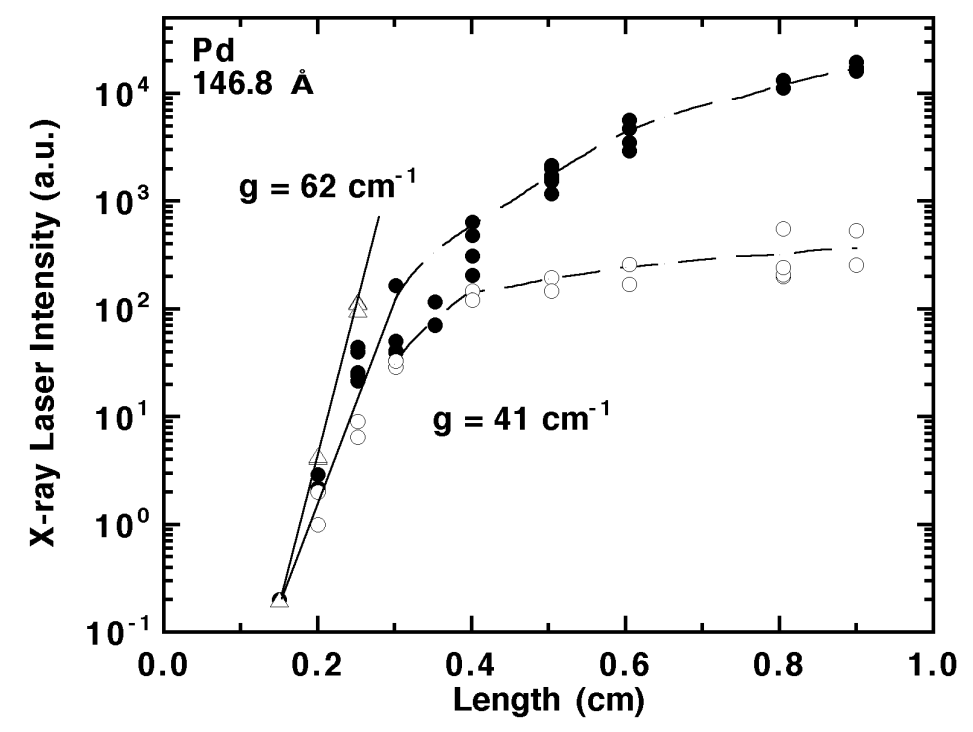

Figure 2. Ni-like Pd intensity versus length plot. Traveling wave (solid circles) and no traveling wave (open circles) for 2 $\mathrm{J}, 600 \mathrm{ps}$ and $5 \mathrm{~J}, 1 \mathrm{ps}$. Traveling wave with higher gain of $62 \mathrm{~cm}^{-1}$ is achieved with $0.5 \mathrm{~J}, 600 \mathrm{ps}$ pulse (open triangles). lines of $\mathrm{Mo}, \mathrm{Pd}$, and $\mathrm{Ag}$, respectively. Overall, the $\mathrm{Pd} \mathrm{x}$-ray laser has the highest intensity, approximately $2-3 \times$ higher than Mo and $4 \times$ higher than the $\mathrm{Ag}$ output. The general trend is decreasing intensity of the $4 d \rightarrow 4 p$ laser line for the highest atomic numbers $\mathrm{Cd}$ and Sn. This is primarily a plasma temperature effect where the short pulse heating is insufficient to strongly collisionally pump the higher excitation energy from the ground state to the upper excited level and drive into saturation.

Figure 2. shows the Pd $146.8 \AA$ $\mathrm{X}$-ray laser intensity versus target length with and without the traveling wave irradiation for $2 \mathrm{~J}, 600 \mathrm{ps}$ and $5 \mathrm{~J}, 1 \mathrm{ps}$ laser energy in the line focus. Using the Linford formula [17], the small signal gain is deduced to be $41 \mathrm{~cm}^{-1}$ for the case with the traveling wave (TW). The output of the $\mathrm{x}$-ray laser smoothly increases to a gain length product of 18.1. Without the traveling wave, the XRL output flattens for target lengths above $0.4 \mathrm{~cm}$ and is $20-$ $100 \times$ lower at $0.9 \mathrm{~cm}$. Also, a higher gain $62 \mathrm{~cm}^{-1}$ was determined for short plasma lengths when a lower energy $\sim 0.5 \mathrm{~J}$ long pulse irradiated the target. 


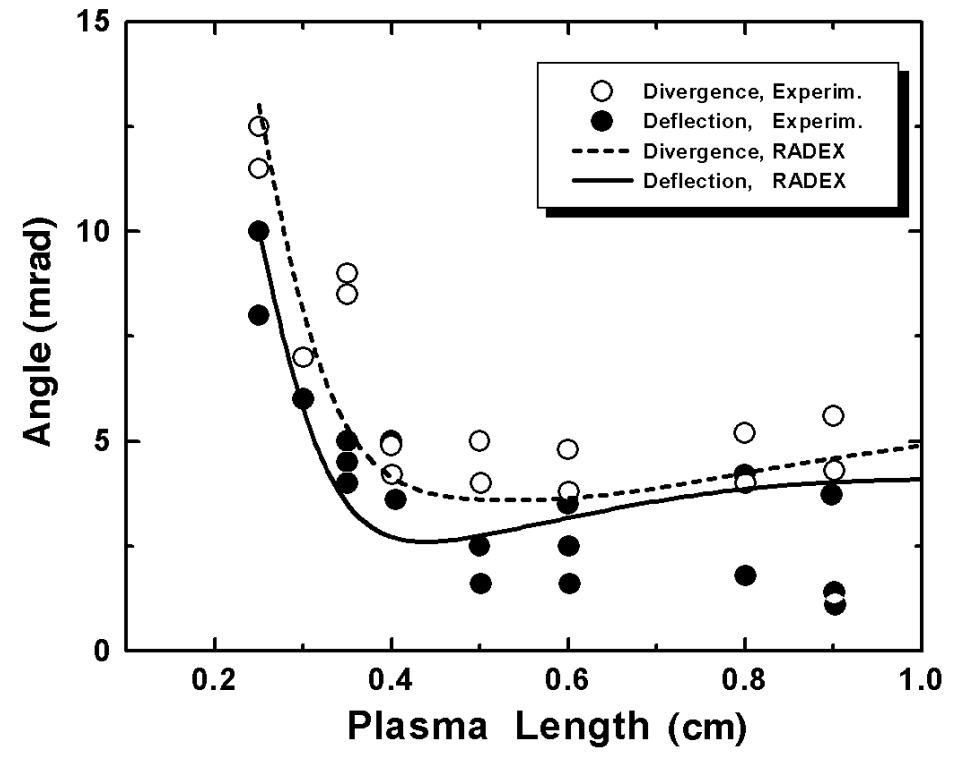

Figure 3. Beam deflection (full circles) and divergence (open circles) of the Pd X-ray laser with plasma length in comparison with RADEX calculations (solid and dash lines, respectively).
One of the main issues regarding the interpretation of experimental data from the transient scheme is being able to identify the characteristics of gain saturation, transient gain lifetime and refraction of the x-ray laser beam out of the high gain region. All of these effects can cause rolloff in the $\mathrm{x}$-ray laser intensity as a function of target length. Our analysis using the RADEX simulation code [8 - 10] concludes that the rolloff in the $\mathrm{x}$-ray laser output without the traveling wave excitation is determined primarily by the short transient gain lifetime in the plasma column and secondly by refraction issues related to density gradients. The traveling wave excitation scheme substantially reduces the former leaving plasma refraction as the most important

effect. In spite of the transient inversion x-ray lasers operating at shorter target lengths than quasisteady state schemes, refraction has a significant influence as a result of the higher gains produced at high densities.

The deflection and beam divergence angles for the Pd laser line are shown as a function of length in Figure 3. For the longest targets the average deflection is $3 \mathrm{mrad}$ away from the target surface with some variation from 1.5 to $5 \mathrm{mrad}$. At lengths below $0.25 \mathrm{~cm}$, beam deflection angles are greater than $10 \mathrm{mrad}$. These rays originate in a very high gain, but dense and highly refractive region. The deflection angle decreases with increasing target length also due to refraction that tends to select the more optimal but less dense areas. There is a slight increase with length reaching an asymptotic value as these rays propagate through the whole active medium. The maximum deflection angle $\left(\mathrm{n}_{\mathrm{e}} / \mathrm{n}_{\mathrm{c}}\right)^{0.5}$ where $\mathrm{n}_{\mathrm{c}}$ is the critical density for this $\mathrm{x}$-ray wavelength, is a reliable tool which reveals the optimal amplification density for Pd ions of $\mathrm{n}_{\mathrm{e}} \sim 0.9 \times 10^{20} \mathrm{~cm}^{-3}$. The divergence also initially decreases with length and finally rebroadens at larger lengths as a consequence of intensity saturation. Similar beam divergence results of $2-4$ mrads are measured for $\mathrm{Cd}$ and $\mathrm{Ag}$ Xray lasers.

The Ni-like Pd x-ray laser saturation intensity can be calculated from numerical simulations of the plasma conditions, where the density, electron and ion temperatures at the time of lasing are $\mathrm{n}_{\mathrm{e}}$ $\sim 0.9 \times 10^{20} \mathrm{~cm}^{-3}, \mathrm{~T}_{\mathrm{e}} \sim 400 \mathrm{eV}$, and $\mathrm{T}_{\mathrm{i}} \sim 80 \mathrm{eV}$, respectively. Values for the stimulated emission cross-section $\sigma_{\text {stim }}=5.2 \times 10^{-15} \mathrm{~cm}^{-2}$ and upper level destruction rate of $\mathrm{A}_{\mathrm{u}}=1.4 \times 10^{12} \mathrm{~s}^{-1}$ are used. The corresponding saturation intensity is $3.7 \times 10^{9} \mathrm{~W} \mathrm{~cm}$. The estimated total uncertainty in this analysis is a factor of two. The experimental $147 \AA$ Pd x-ray laser intensity, $\mathrm{I}_{\text {exp }}$, can be also estimated from the studies of the $188.9 \AA 4 d \rightarrow 4 p$ Mo laser where the laser energy output and gain region dimensions have been measured using a multilayer imaging system [14]. Output energy of 3 $5 \mu \mathrm{J}$ per shot was measured for the Mo x-ray laser after factoring in the recorded signal, mirror reflectivity, filter transmission and CCD quantum efficiency of the system. The relative sensitivity of the spectrometer at different wavelengths was used to convert the integrated $x$-ray laser signal at the spectrometer detector into x-ray laser output energy. The Pd x-ray laser output was determined to be $12 \pm 4 \mu \mathrm{J}$. The gain region of $80 \mu \mathrm{m} \times 50 \mu \mathrm{m}$ and $\mathrm{x}$-ray laser pulse duration of $5-10 \mathrm{ps}$, estimated from previous work (see Osterheld et al. in reference [2]) yield an experimental Pd x-ray laser intensity of $\mathrm{I}_{\exp }=4 \pm 2 \times 10^{10} \mathrm{~W} \mathrm{~cm}^{-2}$ which is $\sim 10$ times higher than $\mathrm{I}_{\text {sat }}$. Due to high small-signal gain the laser output is still increasing non-linearly with length at the longest targets while the laser is operating in the saturation regime. 


\section{NE-LIKE ION SATURATION RESULTS}

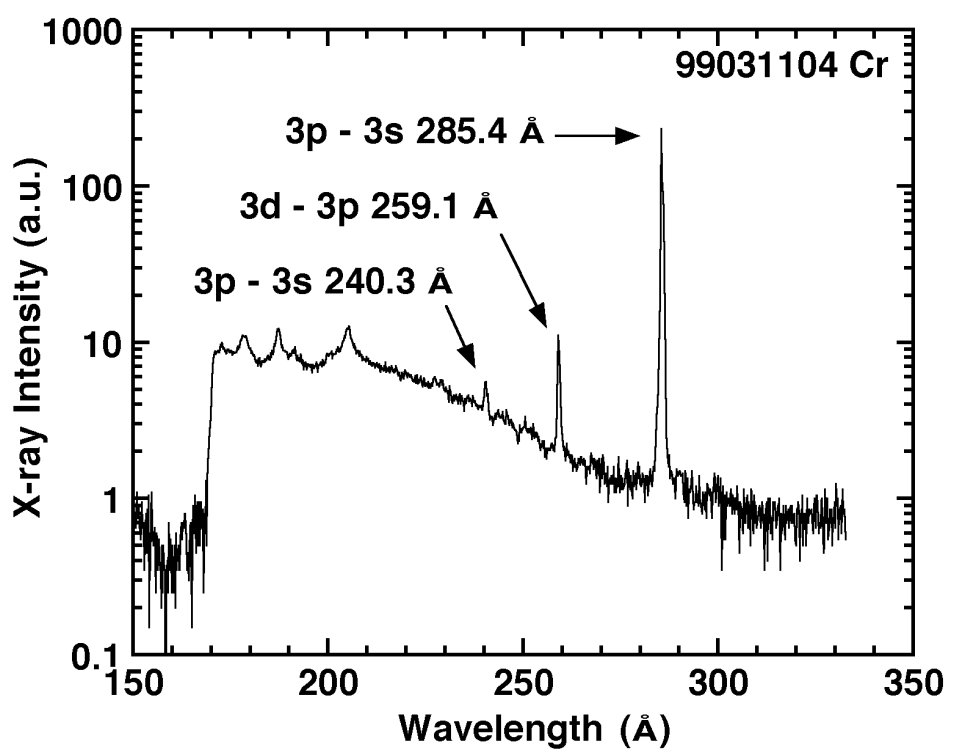

Figure 4. Ne-like $\mathrm{Cr}$ spectrum with TW irradiation incident on 1 $\mathrm{cm}$ target $(3.1 \mathrm{~J}, 600 \mathrm{ps}$ and $4.8 \mathrm{~J}, 1.2 \mathrm{ps})$. Three x-ray laser lines are identified. Al L-edge at $170 \AA$ is visible.

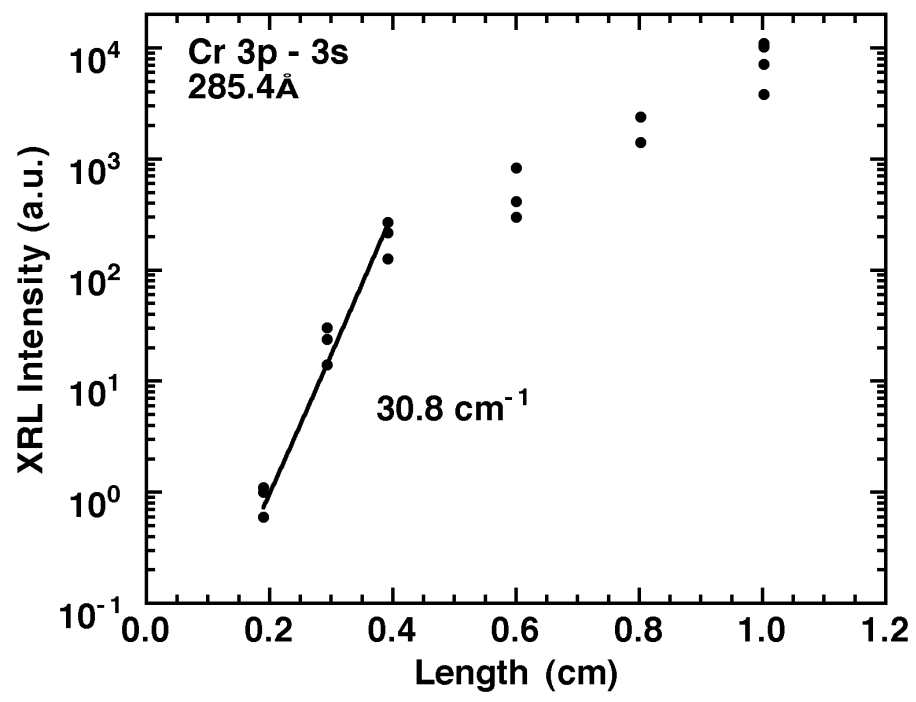

Figure 5. Ne-like Cr $285.4 \AA 3 p \rightarrow 3 s J=0 \rightarrow 1$ intensity versus length plot with TW irradiation.
Previous experiments with lower $Z \mathrm{Ne}$ like ions demonstrated the first transient gain on $\mathrm{Ti}$ with $\sim 10 \mathrm{~J}$ of laser energy on target [9]. Further campaigns with TW irradiation were performed at the RAL Vulcan-CPA laser and generated saturation on Ti at $326 \AA$ and Ge at $196 \AA$ with gain length products of $\sim 17-18$ [11]. These results were achieved with 35 and $60 \mathrm{~J}$ of total laser energy incident on $\mathrm{Ti}$ and Ge targets, respectively. At the same time, experimental studies at LLNL on Ti, V and $\mathrm{Fe}$, without TW irradiation and $\sim 10$ $\mathrm{J}$ of laser energy, showed high gains $\sim 25-55 \mathrm{~cm}^{-1}$ and gain length products of 14.6. While these Ne-like x-ray lasers were close to the predicted saturation intensity and substantially higher than the shorter wavelength Nilike lasers, the issue of saturation was unresolved [18]. It was decided to repeat the experimental studies with the TW irradiation scheme on Ne-like ions of similar $Z$, namely $\mathrm{Cr}(\mathrm{Z}=24)$ and $\mathrm{Fe}$ $(Z=26)$, to determine if saturation was possible with $10 \mathrm{~J}$ laser energy. Preliminary results for Fe targets have been described elsewhere [19], so we will present the $\mathrm{Cr} \mathrm{x}$-ray laser data.

Laser conditions similar to our previous Ne-like ion studies were chosen for irradiating flat slab $\mathrm{Cr}$ targets of length up to $1 \mathrm{~cm}$ : Energy of $0.6-4.8 \mathrm{~J}$ in a $600 \mathrm{ps}$ pulse was followed by a delay of $1.4 \mathrm{~ns}$ before utilizing a $1.2 \mathrm{ps}$ excitation pulse with 5 J. It was found immediately that on longer target lengths the x-ray laser output, with TW excitation, was more

than one order of magnitude higher than previously recorded and required the use of $1-2 \mu \mathrm{m} \mathrm{Al}$ foils placed in front of the spectrometer to attenuate the signal. Fig. 4 shows a typical spectrum from a $1 \mathrm{~cm}$ Cr target irradiated with $3.1 \mathrm{~J}, 600 \mathrm{ps}$ and $4.8 \mathrm{~J}, 1.2 \mathrm{ps}$, total energy less than $8 \mathrm{~J}$. Three $\mathrm{x}$-ray laser lines are identified unambiguously in the spectrum, based on their intensity and narrow beam divergence characteristics. The wavelengths were measured to be $285.4 \AA, 259.1 \AA$ and $240.3 \AA$. The most intense line is the expected $3 p \rightarrow 3 s J=0 \rightarrow 1$ line at $285.4 \AA$ with the $3 d \rightarrow 3 p J=1 \rightarrow 1$ clearly visible at $259.1 \AA$. The population mechanism of the $3 d \rightarrow 3 p$ line for a Ti x-ray laser plasma has been discussed by Nilsen [20]. The weakest lasing line is the short wavelength $3 p \rightarrow 3 s J=0 \rightarrow 1$ line at $240.3 \AA$ previously observed by Li et al. [21]. On a technical note, the Al foil transmission, nominally $1 \mu \mathrm{m}$ thick, was calibrated in situ by taking several identical laser energy shots on target with and without the $\mathrm{Al}$ foil. In this way, line and continuum emission spectra could be recorded and the filter transmission determined from 150 - $330 \AA$ : the foil attenuation at $285.4 \AA$ was determined to be 0.032 . 
An intensity versus length study was performed for target lengths from $0.2 \mathrm{~cm}$ to $1 \mathrm{~cm}$, as shown in Fig. 5. Several shots were repeated for each target length to improve the statistics though overall fewer different target lengths were irradiated than our previous Ni-like Pd measurements. A small-signal gain of $31 \mathrm{~cm}^{-1}$ was determined for targets up to $0.4 \mathrm{~cm}$. The output continues to increase at a lower exponential rate for longer targets giving an overall gain length product of $16.7 \pm$ 0.6 which is above the predicted saturation intensity. As can be seen from Fig. 5 the $\mathrm{Cr} 3 \mathrm{p}-3 \mathrm{~s}$ output is still increasing at $1 \mathrm{~cm}$ and indicates more x-ray laser output can be potentially extracted from the gain medium. Further detailed studies would be required, at longer lengths or higher laser drive intensity, to determine the maximum energy and conversion efficiency for this Ne-like ion.

\section{X-RAY LASER OUTPUT DEPENDENCE WITH SHORT PULSE DURATION}

Table 1. Pre-pulse laser drive parameters for Ni-like Pd, Ag

\begin{tabular}{|c|ccccc|}
\hline Reference & $\begin{array}{l}\mathrm{E} \\
(\mathrm{J})\end{array}$ & $\begin{array}{c}\mathrm{t} \\
(\mathrm{ps})\end{array}$ & $\mathrm{Ipp}_{\mathrm{pp}} / \mathrm{I}$ & $\begin{array}{c}\Delta t \\
(\mathrm{~ns})\end{array}$ & $\begin{array}{c}\mathrm{I} \\
\left(\mathrm{TW} / \mathrm{cm}^{2}\right)\end{array}$ \\
\hline$[4]$ & 150 & 75 & $10 \%$ & 2.2 & 20 \\
\hline$[5]$ & 140 & 100 & $4 \%$ & 3.0 & 80 \\
\hline$[6]$ & 30 & 100 & $10 \%$ & 1.0 & 11 \\
\hline Intermediate ? & 5 & 20 & $0.2 \%$ & 0.7 & 29 \\
\hline
\end{tabular}

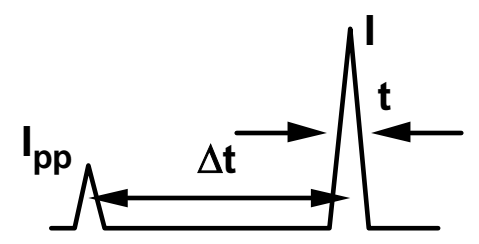

Figure 6. Main parameters of laser drive energy E, pulse duration $\mathrm{t}$, peak intensity $\mathrm{I}$, pulse separation $\Delta \mathrm{t}$, and pre-pulse intensity $I_{p p}$ are shown.
All of the reported transient gain experiments on COMET have been performed with a $1.2-1.5$ ps excitation pulse. Some early experiments in 1997 to investigate the effect on x-ray laser intensity when the short pulse duration was varied from 600 fs to 2 ps were inconclusive. More recently, x-ray laser experiments using 300 fs pulses versus 1.7 ps indicated very strong increases in output when the TW scheme was applied to the shorter pulse [22]. In fact, without the TW irradiation the 300 fs generated no measurable x-ray laser output and so was essential to the $300 \mathrm{fs}$ laser driven scheme. In a related point, many of the $\sim 100$ ps prepulse lasers operating in saturation for $\mathrm{Ni}$ like $\mathrm{Pd}$ and $\mathrm{Ag}$ often have no TW scheme [6] or a quasi-TW scheme $[4,5]$ suggesting that the laser pulse drive is sufficiently longer than gain lifetimes in the plasma

medium for amplification over few centimeter targets. In addition to the substantially longer drives, $75-100 \mathrm{ps}$, the pre-pulse $\mathrm{x}$-ray lasers have also lower peak irradiance, $10^{13}-10^{14} \mathrm{~W} \mathrm{~cm}^{-2}$, than the more intense $\sim 10^{15} \mathrm{~W} \mathrm{~cm}{ }^{-2}$ tabletop picosecond lasers. It is possible that the femtosecond and picosecond laser pulses are over-driving the plasma temperature for creating the population inversion and that some parameter space, of pulse duration and irradiance on target, between the two collisional laser schemes would also be optimal and have good x-ray laser conversion efficiency. Therefore, it seems logical to investigate a tabletop energy $5 \mathrm{~J}$ laser pulse at an intermediate duration of $\sim 20 \mathrm{ps}$ with a traveling wave focused to a modest laser intensity $\sim 3 \times 10^{13} \mathrm{~W} \mathrm{~cm}^{-2}$. Some recent pre-pulse laser parameters are tabulated in Table 1 with Fig. 6 together with the speculative $\sim 20$ ps tabletop driven x-ray laser to illustrate this more clearly. 


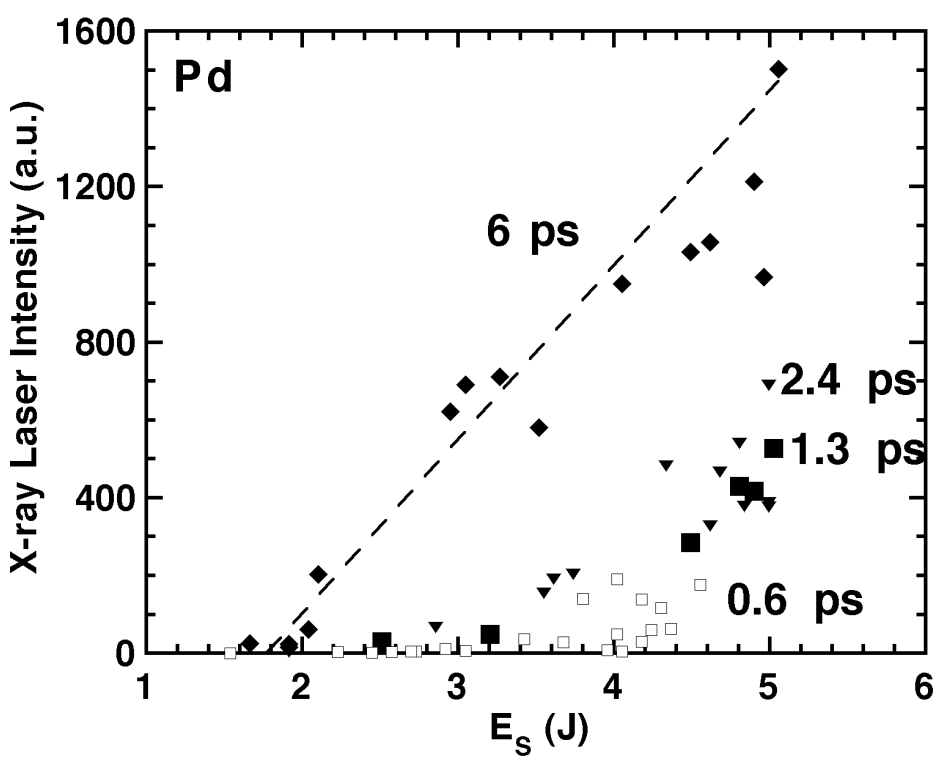

Figure 7. Ni-like Pd $x$-ray laser intensity versus short pulse energy for $0.6 \mathrm{ps}$ (open squares),1.3 ps (solid squares), $2.4 \mathrm{ps}$ (solid triangles) and $6 \mathrm{ps}$ (solid diamonds) data. Dashed line is a guide to the eye through the 6 ps data set.
Laser driver parameters on COMET were chosen similar to the saturated output Ni-like Pd experiments and maintained constant while the short pulse duration, $t_{S}$, was changed [16]. To reiterate, the long pulse energy, $\mathrm{E}_{\mathrm{L}}$, was $0.55-0.85 \mathrm{~J}$ in $600 \mathrm{ps}$, the pulse separation (peak-to-peak) was 700 ps and the laser focus conditions as described in Section 2. The short pulse duration was systematically varied from $0.6-24$ ps (FWHM) by increasing the vacuum compressor grating separation. It should be noted that increasing the compressor grating separation affected the delay between the two laser pulses and that this was compensated to maintain a 700 ps delay. A second order auto-correlator and spectrometer

directly measured the compressed laser pulse duration and spectrum on each shot. The pulse duration was determined to be $0.6,1.3,2.4,6,13,24$ ps giving a $40 \times$ variation in the peak intensity of the short pulse on target. The x-ray laser output was measured as a function of short pulse laser energy, $E_{S}$, for $2-5 \mathrm{~J}$ in the line focus at each pulse duration. It was not possible to increase the pulse duration to $100 \mathrm{ps}$ to directly compare with the pre-pulse experiments listed in Table 1. However, the overall trend is clear from the experimental results plotted in Figures 7 and 8.

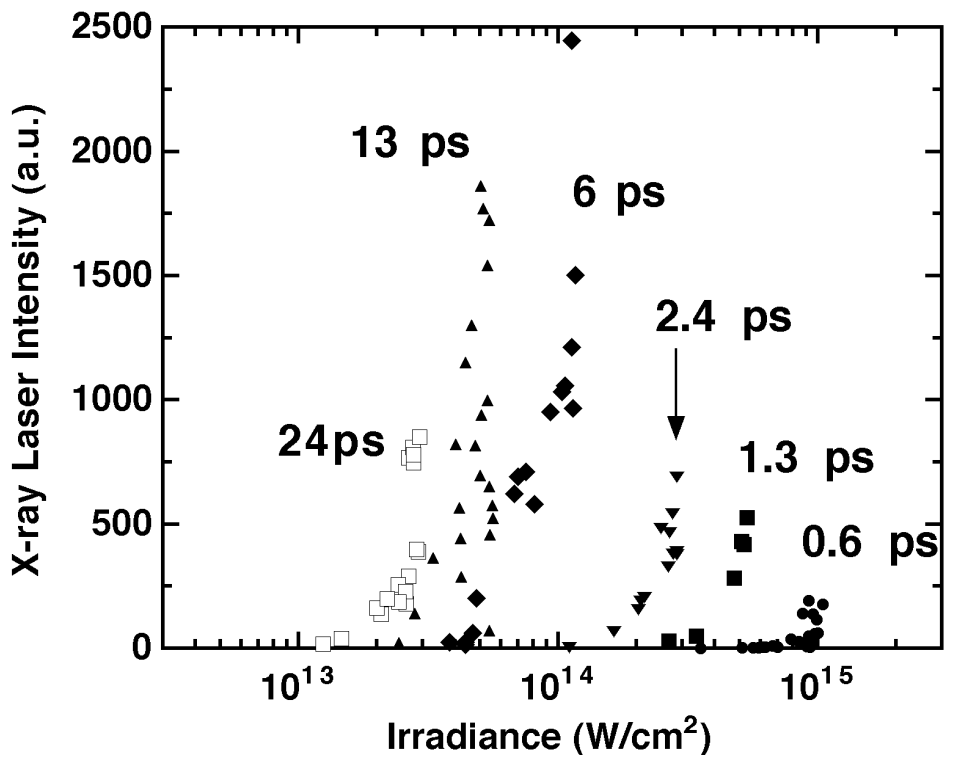

Figure 8. Ni-like Pd x-ray laser intensity versus short pulse irradiance for the six short pulse durations. Maximum intensity for each pulse corresponds to $5 \mathrm{~J}$ in line focus.

Fig. 7 (above) shows x-ray laser output versus short pulse energy for some of the excitation pulse duration data, $0.6,1.3,2.4$ and $6 \mathrm{ps}$, for energy up to $5 \mathrm{~J}$. The $6 \mathrm{ps}$ excitation pulse shows substantially higher output, more than double, than the shorter pulses: the difference between the 2.4 ps and 1.3 ps are within $20-30 \%$ while the $600 \mathrm{fs}$ pulse data set has consistently the lowest overall x-ray laser intensity. The second point to note is the threshold short pulse energy required for lasing does not vary greatly although the laser pulse duration (or irradiance) are changing by $10 \times$. There is, however, some sensitivity of the threshold energy with laser pulse: lowest threshold energy of $\sim 2 \mathrm{~J}$ increases to $3.4 \mathrm{~J}$ when the short pulse duration is decreased from 6 ps to $600 \mathrm{fs}$.

Fig. 8 shows the Ni-like $\mathrm{Pd}$ $146.8 \AA$ line intensity as a function of incident short pulse irradiance for all six pulse durations. As noted in Fig.7, the highest irradiance for each pulse group corresponds to $5 \mathrm{~J}$ energy in the line focus. The nominal 1.3 ps short pulse gives saturated output for $5 \mathrm{~J}$ on target which corresponds to an incident irradiance of $5 \times 10^{14} \mathrm{~W} \mathrm{~cm}^{-2}$. It can be observed that intense lasing is achieved on a range of intensities spanning $10^{13}-10^{15} \mathrm{~W} \mathrm{~cm}^{-2}$, the lower decade overlapping with the pre-pulse experiments listed in Table 1. This can also be stated differently: there exist a large range of pulse durations that generate lasing on the $\mathrm{Pd} 4 d \rightarrow 4 p$ line. It is clear that increasing the pulse duration gives substantially stronger lasing, with the 6 ps and 13 ps pulses having a pronounced $2-5 \times$ 
enhancement for our experimental conditions. In fact these preliminary results would indicate that 10 ps \pm 3 ps drive has the highest efficiency. Increasing the pulse duration further to $24 \mathrm{ps}$, where $5 \mathrm{~J}$ gives $3 \times 10^{13} \mathrm{~W} \mathrm{~cm}^{-2}$ on target, appears to give very similar results to the 2.4 and 1.3 ps drives. However, there is no advantage with a $5 \mathrm{~J}$ drive to using a laser pulse longer than $20 \mathrm{ps}$ at these intensities. It is also clear that there is not an absolute threshold value of irradiance on target that will generate lasing that is independent of laser pulse duration. For example, $10^{14} \mathrm{~W} \mathrm{~cm}^{-2}$ intensity at 2.4 ps is below the threshold for lasing while the same intensity in a 6 ps pulse has saturated output. A direct consequence is that the laser energy absorbed in the plasma is more important, as long as it is above the energy threshold of $2-3 \mathrm{~J}$ for lasing regardless of pulse duration see Fig. 7. These longer excitation pulse conditions also gave repeatable, improved output on the Ag and $\mathrm{Sn}$ x-ray lasers. Further analysis and modeling is underway.

\section{CONCLUSIONS}

$\mathrm{X}$-ray laser results have been described showing the saturation regime for several $\mathrm{Ne}$-like and $\mathrm{Ni}$ like ion collisional schemes by irradiating slab targets with 5-8 J total laser energy in a $600 \mathrm{ps}$ and 1.2 ps laser pulse. A critical feature in the experiment, also shown by other groups, is the utilization of traveling wave irradiation in the short pulse excitation. The x-ray laser output is smoothly increasing at a continually decreasing rate and it should be possible to extract $\sim 5-10 \times$ more energy with a few additional millimeters of target under the present irradiation conditions or by driving the plasma harder. We have also presented preliminary results showing x-ray lasing in saturation for a large range of excitation pulse durations varying from 600 fs to 24 ps while keeping all other laser parameters constant. It was found that maximum x-ray laser output was found for $6 \mathrm{ps}$ to $13 \mathrm{ps}$ pulse durations with $5 \mathrm{~J}$ energy. This corresponds to similar irradiance on target for the pre-pulse experiments but with substantially lower energy in the drive. More significantly, this demonstrates the strong robustness of the transient gain concept where driving table-top x-ray lasers into sub-100 $\AA$ wavelength range will be of primary importance in the future. These experiments and simulations show one of the reliable routes towards this end by creating and utilizing very high gain.

\section{Acknowledgements}

The authors are pleased to acknowledge A. Hazi (LLNL) for continued encouragement and support of this research. Y.L. Li is grateful for support from H. Baldis (ILSA, LLNL). Y.L. Li is presently at the Argonne National Laboratory, Illinois 60439. This work was performed under the auspices of The U.S. Department of Energy by the University of California Lawrence Livermore National Laboratory under contract No. W-7405-Eng-48.

\section{References}

[1] B.J. MacGowan et al., Phys. Fluids B 4, 2326 (1992), A. Carillon et al., Phys. Rev. Lett. 68, 2917 (1992).

[2] X-ray Lasers 1998, ed. Y. Kato, H. Takuma, and H. Daido, IoP Conf. Ser. No. 159 (IoP, Bristol and Philadelphia, 1999), pp720.

[3] J. Nilsen et al., Phys. Rev. A 48, 4682 (1993).

[4] J. Zhang et al., Phys. Rev. Lett. 78(20), 3856 (1997); J.Y. Lin et al., Opt. Commun. 158, 55 (1998); R. Smith et al., Phys. Rev. A 59, R47 (1999).

[5] S. Sebban et al, Phys. Rev. A. 61, 043810 (2000).

[6] R. Tommasini, F. Löwenthal, and J.E. Balmer, Phys. Rev. A 59, 1577 (1999).

[7] J.J. Rocca et al., Phys. Rev. Lett. 77, 1476 (1996); B.R. Benware et al., ibid. 81, 5804 (1998).

[8] Yu.V. Afanasiev and V.N. Shlyaptsev, Sov. J. Quant. Electron. 19, 1606 (1989); V.N. Shlyaptsev et al., Proc. SPIE Int. Soc. Opt. Eng. 2012, 111 (1993).

[9] P.V. Nickles et al., Phys. Rev. Lett. 78(14), 2748 (1997).

[10] J. Dunn et al., Phys. Rev. Lett. 80, 2825 (1998). 
[11] M.P. Kalachnikov et al., Phys. Rev. A 57, 4778 (1998); P.J. Warwick et al., J. Opt. Soc. Am. B 15(6), 1808 (1998).

[12] X-ray Lasers 2000, these proceedings.

[13] J. Dunn, J. Nilsen, A.L. Osterheld, Y. Li, and V.N. Shlyaptsev, Opt. Lett. 24, 101 (1999).

[14] Y. Li, J. Dunn, J. Nilsen, A.L. Osterheld, and V.N. Shlyaptsev, J. Opt. Soc. Am. 17, 1098 (2000).

[15] J.R. Crespo et al, Proc. SPIE Int. Soc. Opt. Eng. 2012, 258 (1993).

[16] J. Dunn et al., Phys. Rev. Lett. 84, 4834 (2000).

[17] G.J. Linford, E.R. Peressini, W.R. Sooy, and M.L. Spaeth, Appl. Opt. 13(2), 379 (1974).

[18] J. Dunn et al., in X-ray Lasers 1998, ed. Y. Kato, H. Takuma, and H. Daido, IoP Conf. Ser. No. 159 (IoP, Bristol and Philadelphia, 1999), pp51.

[19] S. J. Moon et al., SPIE Int. Soc. Opt. Eng. 3776, 9 (1999).

[20] J. Nilsen, Y. L. Li, and J. Dunn, J. Opt. Soc. Am. B 17, 1084 (2000).

[21] Y. Li, G. Pretzler, E.E. Fill, and J. Nilsen, Appl. Phys. B 63, 125 (1996).

[22] A. Klisnick et al., J. Opt. Soc. Am. B 17, 1093 (2000). 